\title{
Correction to: A randomised controlled trial of dietary improvement for adults with major depression (the 'SMILES' trial)
}

\author{
Felice N. Jacka ${ }^{1,4,9,10,13^{*}}$, Adrienne O'Neil ${ }^{1,2,13}$, Rachelle Opie ${ }^{5,13}$, Catherine Itsiopoulos ${ }^{5}$, Sue Cotton ${ }^{3}$, \\ Mohammedreza Mohebbi ${ }^{1}$, David Castle ${ }^{4,11}$, Sarah Dash ${ }^{1,13}$, Cathrine Mihalopoulos ${ }^{7}$, Mary Lou Chatterton ${ }^{7}$, \\ Laima Brazionis ${ }^{5,6}$, Olivia M. Dean ${ }^{1,4,12,13}$, Allison M. Hodge ${ }^{8}$ and Michael Berk ${ }^{1,3,12,13}$
}

\section{Correction}

The original version of this paper [1] did not specify that a website was used in the final year of recruitment, in addition to the other stated recruitment methods. The current version of this website has the title 'Healthy eating as a new approach to treating depression' and can be found here: https://dietdepressionstudy.com/. Twelve participants were recruited after the establishment of this website (June 2014), of whom one reported the website as their recruitment point. In addition to the three media interviews listed on the recruitment website, a fourth interview, which also provided details for recruitment, can be found here: https://www.pressreader.com/australia/geelong-advertiser/20121018/textview

The authors also wish to clarify additional points about their paper. With regards to the information that participants were given about the study hypothesis after recruitment, the Participant Information and Consent form (PICF) stated: "Research has shown that poor diet is closely linked to poor mental health. Improving the quality of a person's diet may lead to better mental health including reduced depression. However, this has not yet been established". Regarding the information that was withheld from the study participants relating to the study hypotheses, this was that no information about content of the diet was provided prior to the study to either group. The potential benefits of social support to mental health were not presented during recruitment, but were discussed with participants when they were given the Participant Information and Consent form (PICF).

\section{Author details}

${ }^{1}$ IMPACT Strategic Research Centre, Deakin University, Geelong, VIC, Australia. ${ }^{2}$ School of Population Health, The University of Melbourne, Melbourne, VIC, Australia. ${ }^{3}$ Orygen, The National Centre of Excellence in Youth Mental Health, Parkville, VIC, Australia. ${ }^{4}$ Department of Psychiatry, University of Melbourne, Melbourne, VIC, Australia. ${ }^{5}$ School of Allied Health, La Trobe University, Melbourne, VIC, Australia. ${ }^{6}$ Department of Medicine, The University of Melbourne, Melbourne, VIC, Australia. ${ }^{7}$ Centre for Population Health Research, Deakin University, Geelong, VIC, Australia. ${ }^{8}$ Cancer Epidemiology and Intelligence Division, Cancer Council Victoria, Carlton, VIC, Australia. ${ }^{9}$ Centre for Adolescent Health, Murdoch Childrens Research Institute, Melbourne, VIC, Australia. ${ }^{10}$ Black Dog Institute, Randwick, NSW, Australia. ${ }^{11}$ St Vincents Hospital, Fitzroy, VIC, Australia. ${ }^{12}$ The Florey Institute of Neuroscience and Mental Health, Parkville, VIC, Australia. ${ }^{13}$ Food \& Mood Centre, Deakin University, IMPACT SRC, School of Medicine, PO Box 281, Geelong, VIC 3220, Australia.

Received: 15 November 2018 Accepted: 15 November 2018 Published online: 28 December 2018

\section{Reference}

1. Jacka FN, et al. A randomised controlled trial of dietary improvement for adults with major depression (the 'SMILES' trial). BMC Medicine. 2017;15:23 https://doi.org/10.1186/s12916-017-0791-y.

\footnotetext{
* Correspondence: f.jacka@deakin.edu.au

'IMPACT Strategic Research Centre, Deakin University, Geelong, VIC, Australia

${ }^{4}$ Department of Psychiatry, University of Melbourne, Melbourne, VIC, Australia

Full list of author information is available at the end of the article
}

(c) The Author(s). 2018 Open Access This article is distributed under the terms of the Creative Commons Attribution 4.0 International License (http://creativecommons.org/licenses/by/4.0/), which permits unrestricted use, distribution, and reproduction in any medium, provided you give appropriate credit to the original author(s) and the source, provide a link to the Creative Commons license, and indicate if changes were made. The Creative Commons Public Domain Dedication waiver (http://creativecommons.org/publicdomain/zero/1.0/) applies to the data made available in this article, unless otherwise stated. 